\title{
Dynamical Analysis of the SEIB Model for Brucellosis Transmission to the Dairy Cows with Immunological Threshold
}

\author{
Wei Zhang, ${ }^{1,2}$ Juan Zhang, ${ }^{1,2}$ Yong-Ping Wu $\mathbb{D}^{3},{ }^{3}$ and Li Li $\mathbb{D}^{2,4}$ \\ ${ }^{1}$ Complex Systems Research Center, Shanxi University, Taiyuan, Shanxi 030006, China \\ ${ }^{2}$ Shanxi Key Laboratory of Mathematical Techniques and Big Data Analysis on Disease Control and Prevention, \\ Shanxi University, Shanxi 030006, China \\ ${ }^{3}$ College of Physics Science and Technology, Yangzhou University, Yangzhou, Jiangsu Province 225002, China \\ ${ }^{4}$ School of Computer and Information Technology, Shanxi University, Shanxi, Taiyuan 030006, China
}

Correspondence should be addressed to Yong-Ping Wu; ypwu@yzu.edu.cn and Li Li; lili831113@sxu.edu.cn

Received 23 February 2019; Revised 22 April 2019; Accepted 28 April 2019; Published 23 May 2019

Academic Editor: Toshikazu Kuniya

Copyright (C) 2019 Wei Zhang et al. This is an open access article distributed under the Creative Commons Attribution License, which permits unrestricted use, distribution, and reproduction in any medium, provided the original work is properly cited.

As we all know, bacteria is different from virus which with certain types can be killed by the immune cells in the body. The brucellosis, a bacterial disease, can invade the body by indirect transmission from environment, which has not been researched by combining with immune cells. Considering the effects of immune cells, we put a minimum infection dose of brucellosis invading into the dairy cows as an immunological threshold and get a switch model. In this paper, we accomplish a thorough dynamics analysis of a SEIB switch model. On the one hand, we can get a disease-free and bacteria-free steady state and up to three endemic steady states which may be thoroughly analyzed in different cases of a minimum infection dose in a switch model. On the other hand, we calculate the basic reproduction number $R_{0}$ and know that the disease-free and bacteria-free steady state is a global stability when $R_{0}<1$, and the one of the endemic steady state is a conditionally global stability when $R_{0}>1$. We find that different amounts of $R_{0}$ may lead to different steady states of brucellosis, and considering the effects of immunology is more serious in mathematics and biology.

\section{Introduction}

Brucellosis is the zoonotic sex contagion which is named as Mediterranean relax heat, wave heat, or wave form heat [13]. It is characterized by extensive host, strong infectivity, and difficulty in radical treatment after infection $[4,5]$. Besides, it has a serious harm to economy, society, and public health. Therefore, brucellosis is listed as one of the communicable diseases that must be notified in the World Organization for Animal Health and is classified as second kind of animal diseases in China. Meanwhile, it is as the first zoonotic disease to be controlled in the National Medium and Long Term Plan for Animal Disease Prevention and Control 2011-2020 formulated by the ministry of agriculture. Brucellosis is acute or chronic infectious disease caused by Brucella which is a group of small bulbous gram-negative bacteria [6, 7]. In 1985, the World Health Organization (WHO) divided Brucella into six species and nineteen biological types [7]. They are more popular with $\mathrm{Br}$. melitensis, Br. brovis, and $\mathrm{Br}$. suis in China, among which the most popular Brucella is Br. melitensis and the second is Br. brovis. The most noticeable symptom of brucellosis is miscarriage in cows and orchitis in bulls. In addition, it can cause arthritis in the knee and wrist frequently $[2,3]$. The route of spread is through direct contact with diseased animals and indirect infection with bacteria in the environment $[8,9]$. And it can be divided into three ways. The first transmitted way is contacting with skin, such as direct contact with droppings, vaginal secretions, and vaginal delivery content of sick animals. It also can be indirectly exposed to the environment and objects contaminated by sick animals. The second infected way is via the digestive tract, like eating food, water, or milk contaminated with pathogens. And the last way of transmission is through the respiratory tract. 
TABLE 1: The description of parameters in original model.

\begin{tabular}{|c|c|c|}
\hline Parameter & Description & Dimension \\
\hline A & The annual introduction number of dairy cows & year ${ }^{-1}$ \\
\hline $\mathrm{q}$ & The annual birth rate of dairy cows & year $^{-1}$ \\
\hline $\mathrm{d}$ & The annual natural elimination rate & year $^{-1}$ \\
\hline$\delta$ & Clinical outcome rate & year $^{-1}$ \\
\hline$c$ & The annual elimination rate for the positive cows & year $^{-1}$ \\
\hline $\mathrm{m}$ & The annual quantity of Brucella & year ${ }^{-1}$ \\
\hline $\mathrm{w}$ & The annual natural mortality of Brucella & year ${ }^{-1}$ \\
\hline$\beta_{1}$ & Cow-to-cow transmission rate & none \\
\hline$\beta_{2}$ & Brucella-to-cow transmission rate & none \\
\hline $\mathrm{h}$ & The sterilizing rate in a disinfection & none \\
\hline e & The number of disinfections every year & once \\
\hline
\end{tabular}

When bacteria enter the body, mainly in the liver, spleen, bone marrow, lymphatic tissue, and other cells, those will grow, multiply, and produce endotoxin which can cause damaged tissues. In the meantime, it often releases bacteria and toxins into the blood causing systemic bacteremia and allergic reactions. But the Brucella does not take effect immediately when it enters the body. The bacteria with a small number will be killed by immune cells and only amounts of bacteria over a certain threshold called a minimum infection dose (MID) in environment can do harm to body [10, 11]. And different bacteria have variant thresholds which should be tested by experiment. Some bacteria threshold can be seen in [12]. Also, there were many authors researching the brucellosis, who had been unconscious of the impact on immunization [13-19]. By referring to [20, 21], we will put the pathogeny of brucellosis and MID into this paper. The authors $[12,20,21]$ incorporated a MID, $c$, into the incidence term $\alpha(B)$, in a cholera transmission model, which was a piecewise continuous function which was zero under the MID and was a Holling - II response curve over that.

$$
\alpha(B)= \begin{cases}0 & \text { if } B<c, \\ \frac{a(B-c)}{(B-c)+H} & \text { if } B \geq c .\end{cases}
$$

where the parameter $c$ represents MID, $H$ is half-saturation pathogen density, and the description of parameter $a$ is maximum rate of infection. One of typical mathematical models given in [22] is as follows:

$$
\begin{aligned}
& \dot{S}=A+q(S+E+I)-\beta_{1} S I-\beta_{2} S V-d S, \\
& \dot{E}=\beta_{1} S I+\beta_{2} S V-d E-\delta E, \\
& \dot{I}=\delta E-d I-c I, \\
& \dot{B}=m(E+I)-(w+e h) B .
\end{aligned}
$$

where all parameters are positive and the description of parameters can be found in Table 1.

In [22], the authors gave a detailed demonstration of the basic reproduction number $R_{0}\left(R_{0}=\left(A(w+e h) \beta_{1} \delta+A m(d+\right.\right.$ $\left.\left.c+\delta) \beta_{2}\right) /(d+c)(d+\delta)(w+e h)(d-q)\right)$, which can estimate the occurrence of the epidemic, and made brief dynamical analysis on the global stability.

Basing on model (2) and considering the immunology of Brucella in the environment, we get a switch system with MID $(M)$. If the number of Brucella in environment $(B)$ is less than or equal to the MID, then we can get a globally asymptotically stable disease-free and bacteria-free steady state while the basic reproduction number is less than or equal to 1 , an unstable disease-free and bacteriafree equilibrium and a conditionally globally stable endemic steady state when the basic reproduction number is more than 1. Else if $B>M$, it is going to be a little bit complicated. Combining with the relationship between parameter $M$ and $M_{g}$ (which is the formula received from the system), we can attain the three different switch systems. One of them can attain four equilibria and can produce a backward bifurcation $[23,24]$ and might be two steady states at most under certain parameter conditions.

\section{Dynamical Analysis of the Model}

2.1. The Dynamical Model. We classify the dairy cows into three compartments: the susceptible compartment $S$, the exposed compartment $E$, and the infectious compartment $I$ in Figure 1. And the Brucella in environment is denoted by $B$.

One of the key differences of (2) is the incidence term $\alpha(B) S$ of indirect transmission of Brucella to susceptible cow [12], where $\alpha(B)$ is the pathogen density dependent component. Unlike $[12,20]$ using Holling - II response curve when the bacteria density is over the MID, a simple linear form is used in this paper. There are chiefly the following reasons. Firstly, the half-saturation pathogen density $(H)$ is difficult to be determined in actual application. Secondly, the Brucella which is discharged to the nature by dairy cows and other livestock is hard to reach saturation. Lastly, that can get more practical conclusions with immunological threshold than [22]. The expression of $\alpha(B)$ in this paper is defined as

$$
\alpha(B)= \begin{cases}0 & \text { if } 0 \leq B \leq M, \\ a(B-M) & \text { if } B>M .\end{cases}
$$




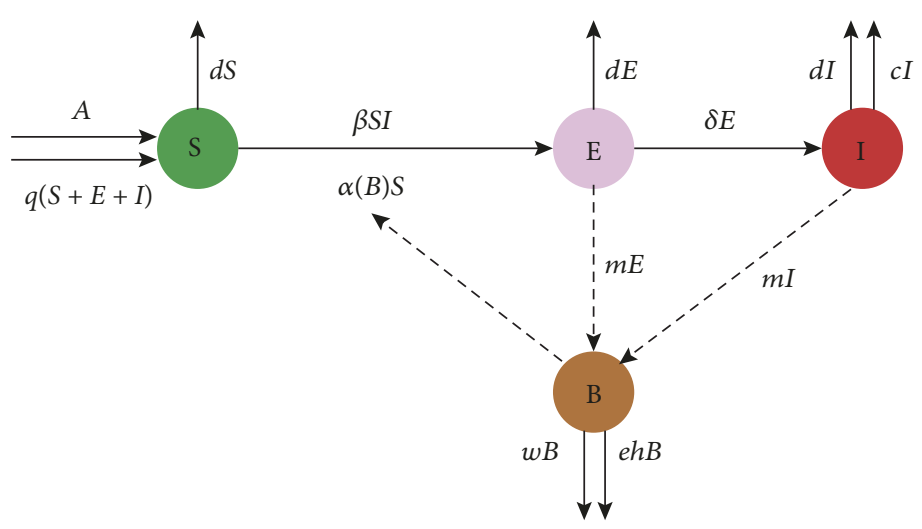

FIGURE 1: A flow diagram of brucellosis transmission.

TABLE 2: The description of new parameters in model (4).

\begin{tabular}{lcc}
\hline Parameter & Description & Dimension \\
\hline $\mathrm{M}$ & MID & cell liter $^{-1}$ \\
$\mathrm{a}$ & Maximum rate of infection & none \\
$\beta$ & Indirect transmission rate & none \\
\hline
\end{tabular}

If the number of bacteria is more than MID, then the Brucella of environment can bring the indirect influence to the dairy cows. At this time, $a$ is representative of maximum rate of indirect infection, and $M$ is denoted MID of Brucella, $B-M$ is representative of bacteria amount which can enter the body and take effect. If the number is less than or equal to MID, then the bacteria of environment have no effect on the susceptible and dairy cows were infected just by the direct transmission from infected to susceptible cow.

So the model is a system of ordinary differential equation as follows:

$$
\begin{aligned}
& \dot{S}=A+q(S+E+I)-\beta S I-d S-\alpha(B) S, \\
& \dot{E}=\beta S I+\alpha(B) S-d E-\delta E, \\
& \dot{I}=\delta E-d I-c I, \\
& \dot{B}=m(E+I)-(w+e h) B,
\end{aligned}
$$

where the description of other parameters which are not mentioned in Table 1 can be found in Table 2 .

Remark 1. In this paper, we also use the condition $d>q$ like [22].

2.2. Forward Invariance. The first equation of (4) gives us that $\dot{S(t)} \geq 0$, when $S=0$. In the same way, we can get $\dot{E(t)} \geq 0$ and $I(t) \geq 0$. Hence, we know that $S(t) \geq 0, E(t) \geq 0$, and $I(t) \geq 0$ for $t>0$. As $\dot{S}+\dot{E}+\dot{I} \leq A-(d-q)(S+E+I)$, we can get $0 \leq S+E+I \leq A /(d-q)$ by using the character of $\dot{S}+\dot{E}+\dot{I}$. Cause $\dot{S}+\dot{E}+\dot{I}=A-(d-q)(S+E+I)$ is a linear function [12]. In the same way, if $B=0$, then $B(t)=0$. Thus, the inequality $B(t) \geq 0$ for $t>0$ is true. If $B(0) \in[0, m(E+I) /(w+e h))$, then $B(t) \in[0, m(E+I) /(w+e h))$ for any $t>0$. To sum up, we get the following theorem.
Theorem 2 (feasible region). The set

$$
\begin{aligned}
\Omega & =\left\{(S, E, I, B): 0 \leq S+E+I \leq \frac{A}{d-q}, 0 \leq B\right. \\
& \left.<\frac{m A}{(w+e h)(d-q)}, S \geq 0, E \geq 0, I \geq 0\right\}
\end{aligned}
$$

defines a forward invariant region of system (2).

\subsection{Equilibria of System (4)}

2.3.1. Equilibria When Bacteria Are Less Than or Equal to $M I D$. When $0 \leq B \leq M$, then $\alpha(B)=0$ and the model is

$$
\begin{aligned}
& \dot{S}=A+q(S+E+I)-\beta S I-d S, \\
& \dot{E}=\beta S I-d E-\delta E, \\
& \dot{I}=\delta E-d I-c I, \\
& \dot{B}=m(E+I)-(w+e h) B .
\end{aligned}
$$

We solve the equation and can get $E_{0}=\left(S_{0}, 0,0,0\right)$, a disease-free and bacteria-free equilibrium where $S_{0}=A /(d-$ $q)$, and a endemic equilibrium $E_{1}=\left(S^{1}, E^{1}, I^{1}, B^{1}\right)$, where

$$
\begin{aligned}
& S^{1}=\frac{(d+\delta)(d+c)}{\beta \delta}, \\
& E^{1}=\frac{(d+c)[\beta \delta A-(d-q)(d+\delta)(d+c)]}{\beta \delta[(d+\delta)(d+c)-q(d+c+\delta)]}, \\
& I^{1}=\frac{\beta \delta A-(d-q)(d+\delta)(d+c)}{\beta[(d+\delta)(d+c)-q(d+c+\delta)]}, \\
& B^{1}=\frac{m(d+c+\delta)[\beta \delta A-(d-q)(d+\delta)(d+c)]}{(w+e h) \beta \delta[(d+\delta)(d+c)-q(d+c+\delta)]} .
\end{aligned}
$$

Remark 3. $S^{1}>0, E^{1}>0, I^{1}>0$ and $B^{1}>0$. 
According to the spectral radius theory $[25,26]$, we can directly obtain the basic reproduction number based on [22] when $\beta_{2}=0$. That is

$$
R_{0}=\rho\left(F V^{-1}\right)=\frac{\beta \delta A}{(d-q)(d+c)(d+\delta)} .
$$

Then we can attain the existence of equilibria: if $R_{0} \leq 1$, then there is only one equilibrium $E_{0}$; if $R_{0}>1$, then there are disease-free and bacteria-free equilibrium $E_{0}$ and endemic equilibrium $E_{1}$.

2.3.2. Equilibria When Bacteria Are More Than MID. When $B>M$, the equation is

$$
\begin{aligned}
& \dot{S}=A+q(S+E+I)-\beta S I-d S-a(B-M) S, \\
& \dot{E}=\beta S I+a(B-M) S-d E-\delta E, \\
& \dot{I}=\delta E-d I-c I, \\
& \dot{B}=m(E+I)-(w+e h) B .
\end{aligned}
$$

Obviously, there is no disease-free and bacteria-free equilibria because of $M \geq 0$. Thus, system (9) implies that

$$
\begin{aligned}
& E=\frac{(d+c)(w+e h) B}{m(d+c+\delta)}, \\
& I=\frac{\delta(w+e h) B}{m(d+c+\delta)}, \\
& S=\frac{(d+\delta) E}{\beta I+\alpha(B)}=\frac{q(E+I)+A}{\beta I+\alpha(B)+d-q} .
\end{aligned}
$$

The equation of $S$ about $B$ can be written as $F(B)=0$. Define $F(B)=B^{2}(w+e h)[(d+c)(d+\delta)-q(d+c+\delta)][\beta \delta(w+$ $e h)+a m(d+c+\delta)]+B m(d+c+\delta)\{-a M(w+e h)[(d+c)(d+$ $\delta)-q(d+c+\delta)]-(w+e h)[\beta \delta A-(d-q)(d+\delta)(d+c)]-$ $a m(d+c+\delta) A\}+a M A m^{2}(d+c+\delta)^{2}$. From the expression, we can know that $F(B)$ is a quadratic function, in which the first and third coefficients are positive. Therefore, the existence of solution for $F(B)=0$ depends on the second coefficient and $\Delta$. If the second coefficient is nonnegative, no matter $\Delta$ is, then $F(B)=0$ has no positive solution. If the second coefficient is negative, the positive solution depends on the sign of $\Delta$ : when $\Delta<0$, there is no solution; when $\Delta=0$, there is one positive solution; and when $\Delta>0$, there are two positive solutions.

Now we simplify them and can get the new second coefficient $b_{1}$ :

$$
\begin{aligned}
b_{1}:= & m(d+c+\delta) \\
& \cdot\left[(-\beta \delta(w+e h)-m a(d+c+\delta)) A+b_{11}\right],
\end{aligned}
$$

where

$$
\begin{aligned}
b_{11}:= & -a M(w+e h)[(d+c)(d+\delta)-q(d+c+\delta)] \\
& +(w+e h)(d-q)(d+\delta)(d+c),
\end{aligned}
$$

and the new $\Delta$ about $A$ :

$$
\begin{aligned}
& \Delta(A):=A^{2} m^{2}(d+c+\delta)^{2}[-\beta \delta(w+e h)-a m(d+c \\
& +\delta)]^{2}+A\left\{-2 m^{2}(d+c+\delta)^{2}(w+e h)\right. \\
& \cdot[\beta \delta(w+e h)+a m(d+c+\delta)] \\
& \cdot[a M[(d+c)(d+\delta)-q(d+c+\delta)] \\
& +(d-q)(d+\delta)(d+c)]\}+m^{2}(d+c+\delta)^{2} \\
& \quad \cdot\{-a M(w+e h)[(d+c)(d+\delta)-q(d+c+\delta)] \\
& +(w+e h)(d-q)(d+\delta)(d+c)\}^{2} .
\end{aligned}
$$

We can know $b_{1}$ is a liner function about $A$ and $\Delta(A)$ is still a quadratic function which the first and third coefficients are positive and the second coefficient is less than zero. Then we calculate the $\Delta(A)$ and can know that the sign of $\Delta(\Delta(A))$ is always positive. So we can solve $\Delta(A)=0$ and get $A_{1}, A_{2}$, where

$$
\begin{aligned}
A_{1} & =(w+e h) \\
& \cdot \frac{a M[(d+c)(d+\delta)-q(d+c+\delta)]+(d-q)(d+c)(d+\delta)-2 \sqrt{a M[(d+c)(d+\delta)-q(d+c+\delta)](d-q)(d+c)(d+\delta)}}{\beta \delta(w+e h)+m a(d+c+\delta)}, \\
A_{2} & =(w+e h) \\
& \cdot \frac{a M[(d+c)(d+\delta)-q(d+c+\delta)]+(d-q)(d+c)(d+\delta)+2 \sqrt{a M[(d+c)(d+\delta)-q(d+c+\delta)](d-q)(d+c)(d+\delta)}}{\beta \delta(w+e h)+m a(d+c+\delta)} .
\end{aligned}
$$

By the analysis with the feature of function, we can get the conclusion as follows.

Proposition 4. The existence of positive solutions for $F(B)=0$ : (i) When $b_{11} \geq 0$ : if $0 \leq A<A_{2}$, then $F(B)=0$ does not get any positive solution; if $A=A_{2}$, then $F(B)=0$ has one positive solution $B_{1}^{+}$; if $A>A_{2}$, then $F(B)=0$ has two positive solutions $B_{1}^{+}, B_{2}^{+}\left(B_{1}^{+}<B_{2}^{+}\right)$. 
TABLE 3

\begin{tabular}{lc}
\hline$F(B)=0$ has one positive solution $B^{+}$ & $F(B)=0$ has two positive solutions $B_{1}^{+}, B_{2}^{+}$ \\
\hline$M \geq 0, A=A_{2}$ & $M \geq 0, A>A_{2}$ \\
\hline$M>\widetilde{M}, A=A_{1}$ & $M>\widetilde{M}, 0 \leq A<A_{1}$ \\
\hline
\end{tabular}

(ii) When $b_{11}<0$ : if $A_{1}<A<A_{2}$, then $F(B)=0$ does not get any positive solution; if $A=A_{1}$ or $A=A_{2}$, then $F(B)=0$ has one positive solution $B^{+}\left(=B_{1}^{+}=B_{2}^{+}\right)$; if $0 \leq A<A_{1}$ or $A>A_{2}$, then $F(B)=0$ has two positive solutions $B_{1}^{+}$, $B_{2}^{+}$.

Note the $\widetilde{M}=(d-q)(d+c)(d+\delta) / a((d+c)(d+\delta)-q(d+c+$ $\delta)$ ) (by the equation of $\left.b_{11}=0\right)$ and we can put Proposition 4 to the chart as shown in Table 3.

It just discusses the existence of positive solutions, but we need consider the value of $M$, where only internal equilibria exist while positive solutions are more than $M$. Hence, we will get conclusions as follows.

Theorem 5. The existence of internal equilibria about $B>$ M:

$$
\begin{aligned}
& A_{f}=\frac{\beta \delta(w+e h)((d+c)(d+\delta)-q(d+c+\delta)) M+m(d+c+\delta)(d-q)(d+c)(d+\delta)}{\beta \delta m(d+c+\delta)}, \\
& A_{g} \\
& =\frac{2 \beta \delta(w+e h)^{2}+a m(w+e h)(d+c+\delta)((d+c)(d+\delta)-q(d+c+\delta)) M+(w+e h) m(d+c+\delta)(d-q)(d+c)(d+\delta)}{m(d+c+\delta)[\beta \delta(w+e h)+m a(d+c+\delta)]} .
\end{aligned}
$$

Proof. We have known the positive value of $B$; now we need combine with the inequality $B>M$. The proof uses character of function $F(B)$.

Firstly, we should know the value of $F(M)$. When $B=M$, we can get $F(M)=\beta \delta(w+e h)^{2}((d+c)(d+\delta)-q(d+c+$ $\delta)) M^{2}-m(d+c+\delta)(w+e h)(\beta \delta A-(d-q)(d+c)(d+\delta)) M$, and the relationship between $F(M)$ and 0 is translated into the relationship between $A$ and $A_{f}$ :

(1) When $M>0$ : if $A>A_{f}$, then $F(M)<0$, if $A=A_{f}$, then $F(M)=0$, and if $A<A_{f}$, then $F(M)>0$.

(2) When $M=0$, we can get $F(M)=0$.

Secondly, we need to know position of $M$ and symmetry axis of $F(M)$. We put them into the formula $g(M)$ := $\left[2 \beta \delta(w+e h)^{2}+a m(w+e h)(d+c+\delta)\right]((d+c)(d+\delta)-$ $q(d+c+\delta)) M-m(d+c+\delta)[(w+e h)(\beta \delta A-(d-q)(d+$ $c)(d+\delta))+m a(d+c+\delta) A]$. In the same way, we translate them into the relationship between $A$ and $A_{g}$. It means that when $M \geq 0$ : if $A>A_{g}$, then $g(M)<0$ ( $M$ is on the left of axis of symmetry); if $A=A_{g}$, then $g(M)=0$ ( $M$ is the axis of symmetry); and if $A<A_{g}$, then $g(M)>0$ ( $M$ is on the right of axis of symmetry).

Thirdly, we calculate the relationship of $A_{f}, A_{g}, A_{1}, A_{2}$. Then we can get the relationships among them as follows:
(1) If $M>0$ :

(i) when $A>A_{f}$, there is an internal equilibrium $E_{2}^{+}$;

(ii) when $A=A_{f}>A_{g}$, there is an internal equilibrium $E_{2}^{+}$;

(iii) when $\max \left\{A_{g}, A_{2}\right\}<A<A_{f}$, there are two internal equilibria $E_{1}^{+}, E_{2}^{+}$;

(iv) when $A=A_{2}>A_{g}$, there is an internal equilibrium $E^{+}$;

(2) If $M=0$ and $A>A_{2}=A_{g}$, then there is an internal equilibrium $E_{2}^{+}$.

where 
TABLE 4

\begin{tabular}{|c|c|c|c|c|}
\hline$F(B)$ & $F(M)$ & $g(M)$ & Conclusions & Solutions \\
\hline$M \geq 0, A=A_{2}$ & - & $M \geq 0, A>A_{g}$ & $M>0, A=A_{2}>A_{g}$ & $B^{+}$ \\
\hline \multirow{4}{*}{$M \geq 0, A>A_{2}$} & $M>0, A>A_{f}$ & - & $M>0, A>A_{f}$ & $B_{2}^{+}$ \\
\hline & $M=0$ & $M \geq 0, A>A_{g}$ & $M=0, A>A_{2}=A_{g}$ & $B_{2}^{+}$ \\
\hline & $M>0, A=A_{f}$ & $M \geq 0, A>A_{g}$ & $M>0, A=A_{f}>A_{g}$ & $B_{2}^{+}$ \\
\hline & $M>0, A<A_{f}$ & $M \geq 0, A>A_{g}$ & $M>0, \max \left\{A_{2}, A_{g}\right\}<A<A_{f}$ & $B_{1}^{+}, B_{2}^{+}$ \\
\hline
\end{tabular}

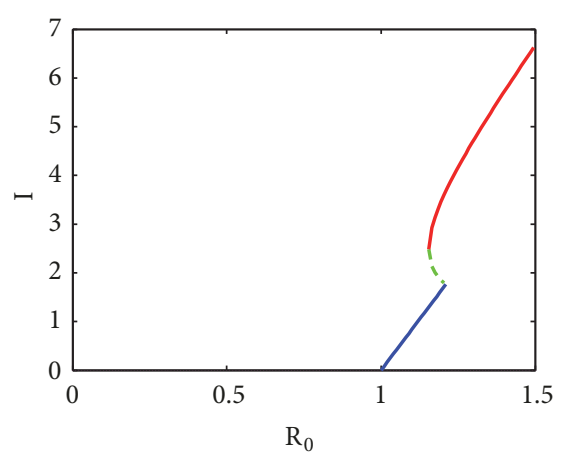

(a) $P_{2}>1$

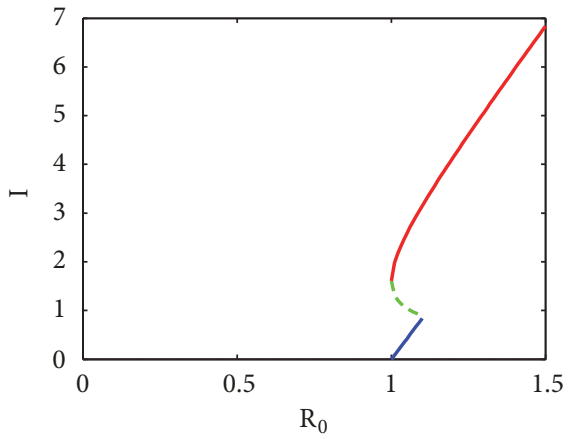

(b) $P_{2}=1$

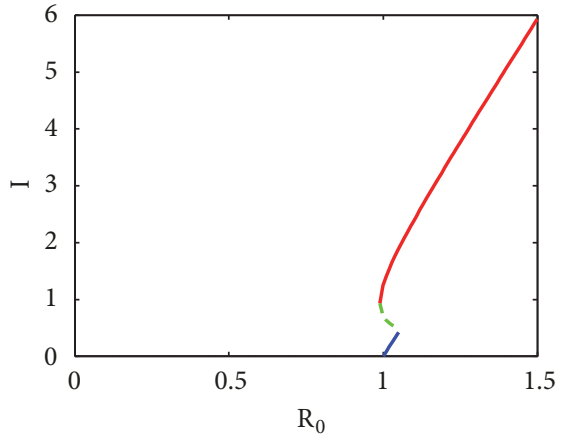

(c) $P_{2}<1$

Figure 2: Density of the infected dairy cows as a function of the basic reproduction number with $0<M<M_{g}$. The common values of (a), (b), and (c) used for the parameters are as follows: $w=3, d=2, c=0.8, \delta=4, q=1, \beta=0.2$, and $a=2$. The different values are (a) $m=0.1$, $M=0.1$; (b) $m=0.0932, M=0.0468$; and (c) $m=0.05, M=0.0135$. The forward bifurcation occurs at $R_{0}=1$ in all the pictures and the backward bifurcation occurs at $R_{0}=1.2101$ in picture (a), at $R_{0}=1.1056$ in picture (b) and at $R_{0}=1.0567$ in picture (c).

Theorem 7 (existence of equilibria in Figures 2 and 3). The equilibrium $E_{0}=\left(S_{0}, 0,0,0\right)$ always exists in $\Omega$. Here are the existences of internal equilibria:

(1) If $0<M<M_{g}$ :

(i) If $P_{2}>1$ : when $1<R_{0}<P_{2}$, there only exists $E_{1}$; when $R_{0}=P_{2}$, there exist $E_{1}, E^{+}$; when $P_{2}<$ $R_{0}<P_{f}$, there exist $E_{1}, E_{1}^{+}, E_{2}^{+}$; when $R_{0}=P_{f}$, there exist $E_{1}, E_{2}^{+}$; when $R_{0}>P_{f}$, there exists $E_{2}^{+}$.

(ii) If $P_{2}=1$ : when $R_{0}=P_{2}$, there exists $E^{+}$; when $1<R_{0}<P_{f}$, there exist $E_{1}, E_{1}^{+}, E_{2}^{+}$; when $R_{0}=$ $P_{f}$, there exist $E_{1}, E_{2}^{+}$; when $R_{0}>P_{f}$, there exists $E_{2}^{+}$.

(iii) If $P_{2}<1$ : when $R_{0}=P_{2}$, there exists $E^{+}$; when $P_{2}<R_{0} \leq 1$, there exist $E_{1}^{+}, E_{2}^{+}$; when $1<R_{0}<$ $P_{f}$, there exist $E_{1}, E_{1}^{+}, E_{2}^{+}$; when $R_{0}=P_{f}$, there exist $E_{1}, E_{2}^{+}$; when $R_{0}>P_{f}$, there exists $E_{2}^{+}$.

(2) If $M \geq M_{g}$ : when $1<R_{0} \leq P_{f}$, there exists $E_{1}$; when $R_{0}>P_{f}$, there exists $E_{2}^{+}$.

(3) If $M=0$ (in this case, $P_{f}=1$.): when $R_{0}>P_{2}$, there exists $E_{2}^{+}$.

Theorem 7 describes the existence of equilibria under the different parameters condition (Table 5), where disease-free and bacteria-free equilibrium $E_{0}$ always exists. Besides, we give the stability of the equilibria, which will be proved in next part. We just give the stability of $E_{0}$ and $E_{1}$; others are too difficult to give the strict mathematical proofs because of lacking exact expression. Therefore, the local stability of $E_{1}^{+}$ and $E_{2}^{+}$will be demonstrated numerically in Section 4 .

\section{Stability of Equilibria}

3.1. Local Stability of $E_{0}$ and $E_{1}$. We calculate the Jacobian to analyze the local stability of each of the equilibria. When $0 \leq B \leq M$, the Jacobian is

$$
\begin{aligned}
& J(S, E, I, B) \\
& =\left(\begin{array}{cccc}
q-d-\beta I & q & q-\beta S & 0 \\
\beta I & -(d+\delta) & \beta S & 0 \\
0 & \delta & -(d+c) & 0 \\
0 & m & m & -(w+e h)
\end{array}\right) .
\end{aligned}
$$

Now considering $E_{0}$, we use $\lambda$ for eigenvalues. We can compute

$$
\begin{aligned}
\operatorname{det}\left(\lambda I-J_{E_{0}}\right)= & {[\lambda-(q-d)](\lambda+w+e h) } \\
& \cdot\left[(\lambda+d+\delta)(\lambda+d+c)-\beta \delta S_{0}\right] .
\end{aligned}
$$

Note $h(\lambda)=(\lambda+d+\delta)(\lambda+d+c)-\beta \delta S_{0}$. We can find that $\lambda_{1}=-(d-q)<0, \lambda_{2}=-(w+e h)<0$. Now we just consider the eigenvalues of $h(\lambda)$. The Routh - Hurwitz coefficients of the $h(\lambda)$ are

$$
\begin{aligned}
& \Delta_{1}=d+c+d+\delta>0 \\
& \Delta_{2}=(d+c+d+\delta)\left[(d+\delta)(d+c)-\beta \delta S_{0}\right] .
\end{aligned}
$$


TABLE 5: Existence of endemic equilibria of model (4).

\begin{tabular}{|c|c|c|c|}
\hline Condition 1 & Condition 2 & Condition 3 & Endemic equilibria \\
\hline \multirow[t]{14}{*}{$0<M<M_{g}$} & $P_{2}>1$ & $1<R_{0}<P_{2}$ & $E_{1}$ \\
\hline & & $R_{0}=P_{2}$ & $E_{1}, E^{+}$ \\
\hline & & $P_{2}<R_{0}<P_{f}$ & $E_{1}, E_{1}^{+}, E_{2}^{+}$ \\
\hline & & $R_{0}=P_{f}$ & $E_{1}, E_{2}^{+}$ \\
\hline & & $R_{0}>P_{f}$ & $E_{2}^{+}$ \\
\hline & $P_{2}=1$ & $R_{0}=P_{2}$ & $E^{+}$ \\
\hline & & $1<R_{0}<P_{f}$ & $E_{1}, E_{1}^{+}, E_{2}^{+}$ \\
\hline & & $R_{0}=P_{f}$ & $E_{1}, E_{2}^{+}$ \\
\hline & & $R_{0}>P_{f}$ & $E_{2}^{+}$ \\
\hline & $P_{2}<1$ & $R_{0}=P_{2}$ & $E^{+}$ \\
\hline & & $P_{2}<R_{0} \leq 1$ & $E_{1}^{+}, E_{2}^{+}$ \\
\hline & & $1<R_{0}<P_{f}$ & $E_{1}, E_{1}^{+}, E_{2}^{+}$ \\
\hline & & $R_{0}=P_{f}$ & $E_{1}, E_{2}^{+}$ \\
\hline & & $R_{0}>P_{f}$ & $E_{2}^{+}$ \\
\hline \multirow[t]{2}{*}{$M \geq M_{g}$} & - & $1<R_{0} \leq P_{f}$ & $E_{1}$ \\
\hline & & $R_{0}>P_{f}$ & $E_{2}^{+}$ \\
\hline$M=0$ & - & $R_{0}>P_{2}$ & $E_{2}^{+}$ \\
\hline
\end{tabular}

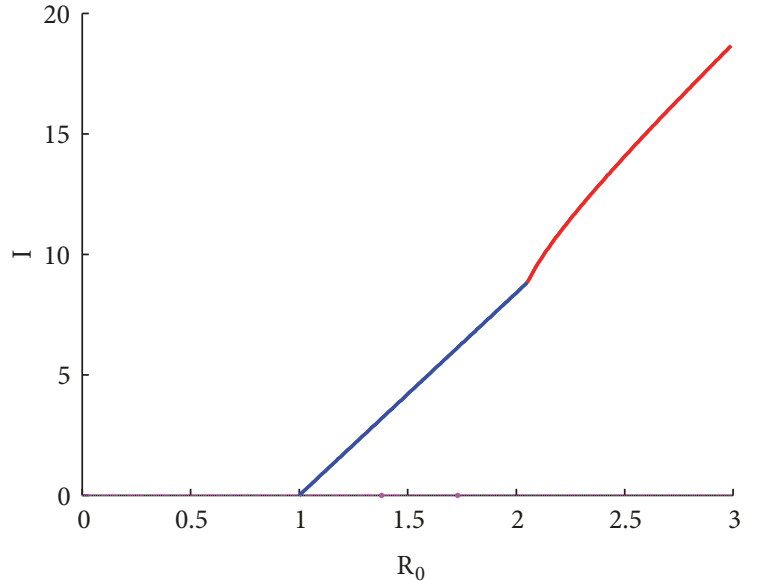

(a) $M \geq M_{g}$

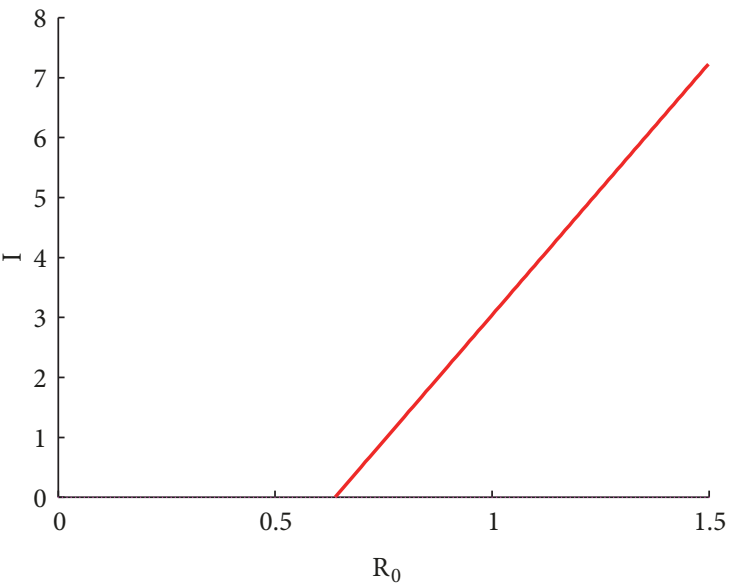

(b) $M=0$

Figure 3: The relationship between the basic reproduction number and infected dairy cows while $M \geq M_{g}$ and $M=0$, where (2) and (3) of Theorem 9 holds. The common values of (a) and (b) used for the parameters are as follows: $w=3, d=2, c=0.8, \delta=4, q=1, \beta=0.2, a=2$, and $m=0.1$. The different values of (a) and (b) are (a) $M=0.5$, (b) $M=0$. There is a forward bifurcation at $R_{0}=1$ in (a). The bifurcation at $R_{0}=0.6383$ in (b) is still forward.

If $R_{0}<1$, then $\Delta_{2}>0$; if $R_{0}>1$, then $\Delta_{2}<0$. Thus the eigenvalues of $h(\lambda)$ are negative when $R_{0}<1$. So the diseasefree and bacteria-free equilibria $E_{0}$ are locally asymptotically stable while $R_{0}<1$. If $R_{0}>1$, then $E_{0}$ is a saddle-point equilibrium.

Now considering $E_{1}$. The characteristic polynomial of Jacobian of $E_{1}$ is

$$
\operatorname{det}\left(\lambda I-J_{E_{1}}\right)=a_{0} \lambda^{3}+a_{1} \lambda^{2}+a_{2} \lambda+a_{3}=0,
$$

where

$$
\begin{aligned}
a_{0}= & 1, \\
a_{1}= & \frac{\beta \delta A-q(d-q)(d+c+\delta)}{(d+\delta)(d+c)-q(d+c+\delta)} \\
& +(d+c+d+\delta), \\
a_{2}= & \frac{\beta \delta A-[q(d-q)(d+c+\delta)](d+\delta+d+c)}{(d+\delta)(d+c)-q(d+c+\delta)}, \\
a_{3}= & \beta \delta A-(d-q)(d+\delta)(d+c) .
\end{aligned}
$$


The Routh - Hurwitz coefficients of the $\operatorname{det}\left(\lambda I-J_{E_{0}}\right)$ are

$$
\begin{aligned}
& \Delta_{1}=a_{1}, \\
& \Delta_{2}=a_{1} a_{2}-a_{3}, \\
& \Delta_{3}=a_{3}\left(a_{1} a_{2}-a_{3}\right) .
\end{aligned}
$$

where $a_{1}>(\beta \delta A-q(d-q)(d+c+\delta)) /((d+\delta)(d+c)-q(d+c+$ $\delta))>(\beta \delta A-(d-q)(d+\delta)(d+c)) /((d+\delta)(d+c)-q(d+c+\delta))>0$ and $a_{3}>0$, when $R_{0}>1$.

As for the condition $a_{1} a_{2}-a_{3}$, we have the following expression:

$$
a_{1} a_{2}-a_{3}=h_{1}+h_{2}+h_{3}
$$

where

$$
\begin{aligned}
& h_{1}=\frac{[\beta \delta A-q(d-q)(d+c+\delta)]\{[\beta \delta A-q(d-q)(d+c+\delta)](d+c+d+\delta)-[q \beta \delta A-q(d-q)(d+c)(d+\delta)]\}}{[(d+c)(d+\delta)-q(d+c+\delta)]^{2}}, \\
& h_{2}=\frac{[\beta \delta A-q(d-q)(d+c+\delta)](d+c+d+\delta)^{2}-(d+c+d+\delta)[q \beta \delta A-q(d-q)(d+c)(d+\delta)]}{(d+c)(d+\delta)-q(d+c+\delta)}, \\
& h_{3}=-[\beta \delta A-(d-q)(d+\delta)(d+c)] .
\end{aligned}
$$

Now, we magnify them:

$h_{1}$

$$
\begin{aligned}
& >\frac{[(d-q)(d+c)(d+\delta)-q(d-q)(d+c+\delta)]\{[\beta \delta A-q(d-q)(d+c+\delta)](d+c+d+\delta)-[q \beta \delta A-q(d-q)(d+c)(d+\delta)]\}}{[(d+\delta)(d+c)-q(d+c+\delta)]^{2}} \\
& =\frac{(d-q)\{[\beta \delta A-q(d-q)(d+c+\delta)](d+c+d+\delta)-q[\beta \delta A-(d-q)(d+c)(d+\delta)]\}}{(d+\delta)(d+c)-q(d+c+\delta)}, \\
& h_{1}+h_{2} \\
& >\frac{\{[\beta \delta A-q(d-q)(d+c+\delta)](d+c+d+\delta)(d+\delta+d+c+d-q)-[q \beta \delta A-q(d-q)(d+c)(d+\delta)](d+\delta+d+c+d-q)\}}{(d+\delta)(d+c)-q(d+c+\delta)}, \\
& h_{1}+h_{2}+h_{3} \\
& >\frac{[\beta \delta A-q(d-q)(d+c+\delta)](d+c+d+\delta)(d+\delta+d+c+d-q)-[\beta \delta A-(d-q)(d+c)(d+\delta)]\left[2 d q-q^{2}+(d+c)(d+\delta)\right]}{(d+\delta)(d+c)-q(d+c+\delta)} \\
& >\frac{[\beta \delta A-(d-q)(d+c)(d+\delta)](d+c+d+\delta)(d+c+d+\delta+d-q)-[\beta \delta A-(d-q)(d+c)(d+\delta)]\left[2 d q-q^{2}+(d+c)(d+\delta)\right]}{(d+\delta)(d+c)-q(d+c+\delta)} \\
& >\frac{[\beta \delta A-(d-q)(d+c)(d+\delta)]\left[(d+c)^{2}+(d+\delta+d-q)(d+\delta+d+c)+q^{2}-2 d^{2}\right]}{(d+\delta)(d+c)-q(d+c+\delta)} .
\end{aligned}
$$

We know that $d>q$, so $(d+c)(d+\delta)>q(d+c+\delta)$ and then $a_{1} a_{2}-a_{3}>0$. Hence, the Routh-Hurwit $z$ conditions are satisfied when $R_{0}>1$ and $E_{1}$ is locally asymptotically stable.

Theorem 8 (local stability). System (4) has equilibria as follows:

When $0 \leq B \leq M$ and $R_{0}<1$, there is only one equilibrium $E_{0}$ which is locally asymptotically stable. When $0 \leq B \leq M$ and $R_{0}>1, E_{0}$ is unstable and a unique endemic equilibrium $E_{1}$ exists which is locally asymptotically stable.
We study the stability of equilibria in order to explain more epidemiological implications. Taking Figure 2(a) as an example, we can get the following biological significance. If $E_{0}$ is stable $\left(R_{0}<1\right)$, the brucellosis will fade as time goes by. If $E_{1}$ is stable $\left(1<R_{0}<P_{2}\right)$, disease will maintain the level of $I_{1}$ all the time. With the scope of $R_{0}\left(P_{2}<R_{0}<P_{f}\right)$, disease will continue at low-level state $\left(I_{1}\right)$ when the current status is in the attraction domain of $E_{1}$ and will be going to stay at high-level state $\left(I_{2}^{+}\right)$while the current status exactly situate in the attraction domain of $E_{2}^{+}$, which will be demonstrated 
numerically in Section 4. Similarly, disease will be going to stay at the level of $I_{2}^{+}$only if $R_{0}>P_{f}$ is true.

3.2. Global Stability of $E_{0}$ and $E_{1}$. In this section, we will demonstrate the global stability of the endemic equilibrium of system (4) by using the Lyapunov function [27, 28].

Theorem 9. If $0 \leq B \leq M$ and $R_{0}<1$, then $E_{0}$ is globally asymptotically stable in $\Omega$.

Proof. As we can see, $B$ is not acting on the other subclasses. So we can define the Lyapunov function

$$
V(S, E, I, B)=S-S_{0}-S_{0} \ln \frac{S}{S_{0}}+E+\frac{d+\delta}{\delta} I .
$$

Then the derivative of $V$ along system (6) is

$$
\begin{aligned}
\frac{d V}{d t}= & \left(1-\frac{S_{0}}{S}\right)[A-(d-q) S+q(E+I)-\beta S I] \\
& +[\beta S I-(d+\delta) E]+\frac{d+\delta}{\delta}[\delta E-(d+c) I] \\
= & \left(1-\frac{S_{0}}{S}\right)[A-(d-q) S+q(E+I)]+\beta S_{0} I \\
& -(d+\delta) E+\frac{d+\delta}{\delta}[\delta E-(d+c) I] \\
= & \left(1-\frac{S_{0}}{S}\right)[A-(d-q) S+q(E+I)] \\
& +I \frac{\beta \delta A-(d-q)(d+c)(d+\delta)}{\delta(d-q)} .
\end{aligned}
$$

According to the definition of the invariant region, we can know that $S \leq S_{0}$. So $d V / d t<0$ is true and $d V / d t=0$ holds only if $S=S_{0}$, when $R_{0}<1 . d V / d t=0$ holds only if $S=S_{0}$ when $R_{0}=1$. Thus the disease-free and bacteria-free equilibrium $E_{0}$ is globally asymptotically stable by LaSalle's Invariance Principle [29].

Theorem 10. If $0 \leq B \leq M, R_{0}>1$, and $q=0$, then $E_{1}$ is a conditionally globally asymptotically stable in $\Omega$.

Proof. In this proof, we use the same way to demonstrate the stability when $q=0$. Similarly, $B$ is not acting on the other subclasses, so we define the Lyapunov function as follows:

$$
\begin{aligned}
V(S, E, I, B)= & S-S^{1}-S^{1} \ln \frac{S}{S^{1}}+E-E^{1}-E^{1} \ln \frac{E}{E^{1}} \\
& +\frac{d+\delta}{\delta}\left(I-I^{1}-I^{1} \ln \frac{I}{I^{1}}\right) .
\end{aligned}
$$

Then the derivative of $V$ along system (6) is

$$
\begin{aligned}
\frac{d V}{d t}= & \left(1-\frac{S^{1}}{S}\right)[A-d S-\beta S I] \\
& +\left(1-\frac{E^{1}}{E}\right)[\beta S I-(d+\delta) E] \\
& +\frac{d+\delta}{\delta}\left(1-\frac{I^{1}}{I}\right)[\delta E-(d+c) I] .
\end{aligned}
$$

Let $s=S / S^{1}, e=E / E^{1}$, and $i=I / I^{1}$. Then we can get

$$
\begin{aligned}
A-d S^{1}-\beta S^{1} I^{1} & =0, \\
\beta S^{1} I^{1}-(d+\delta) E^{1} & =0, \\
\delta E^{1}-(d+c) I^{1} & =0 .
\end{aligned}
$$

Combining with them, we can obtain that

$$
\begin{aligned}
\frac{d V}{d t}= & S^{1}\left(2 d-d s-\frac{d}{s}\right)+\beta S^{1} I^{1}\left(1-\frac{1}{s}+i-\frac{s i}{e}\right) \\
& +E^{1}\left[(d+\delta)-\frac{(d+\delta) e}{i}\right] \\
& +I^{1}\left[-\frac{(d+\delta)(d+c) i}{\delta}+\frac{(d+c)(d+\delta)}{\delta}\right] \\
= & d S^{1}\left(2-s-\frac{1}{s}\right)+\beta S^{1} I^{1}\left(3-\frac{1}{s}-\frac{s i}{e}-\frac{e}{i}\right) .
\end{aligned}
$$

Combining with inequality of arithmetic and geometric means, we can know $2-s-1 / s \leq 0$ and $(3-1 / s-s i / e-e / i) \leq 0$. Thus,

$$
\frac{d V}{d t} \leq 0 .
$$

According to the definition of the invariant region, only if $s=e=i=1$ means that $S=S^{1}, E=E^{1}$ and $I=I^{1}$, then $d V / d t=0$. Similarly with Theorem 9 , we can get the endemic equilibrium $E_{1}$ which is globally asymptotically stable by LaSalle's Invariance Principle in $\Omega$ when $q=0$.

\section{Numerical Results}

In an epidemic model, the basic reproduction number $R_{0}$ is calculated and verified to be a threshold for the dynamics of the disease. The ultimate objective is to control the disease by making the basic reproduction number $R_{0}$. In the previous analysis of this paper, we analyze the different equilibria in variant ranges of $R_{0}$. Now, we should know the final infectious size of dairy cows depending on the $R_{0}$.

In Figure 4(a), only if $R_{0}<\min \left\{1, P_{2}\right\}$ (Figures 2(a), 2(b), 2(c), 3(a), and 3(b)), we can know that the number of infected dairy cows will obtain the same disease-free and bacteria-free equilibrium $E_{0}$ no matter how much the initial values are and the relationship about $M, M_{g}$, and 0 is. In biology, this means only if the basic reproduction number demands the formula, 


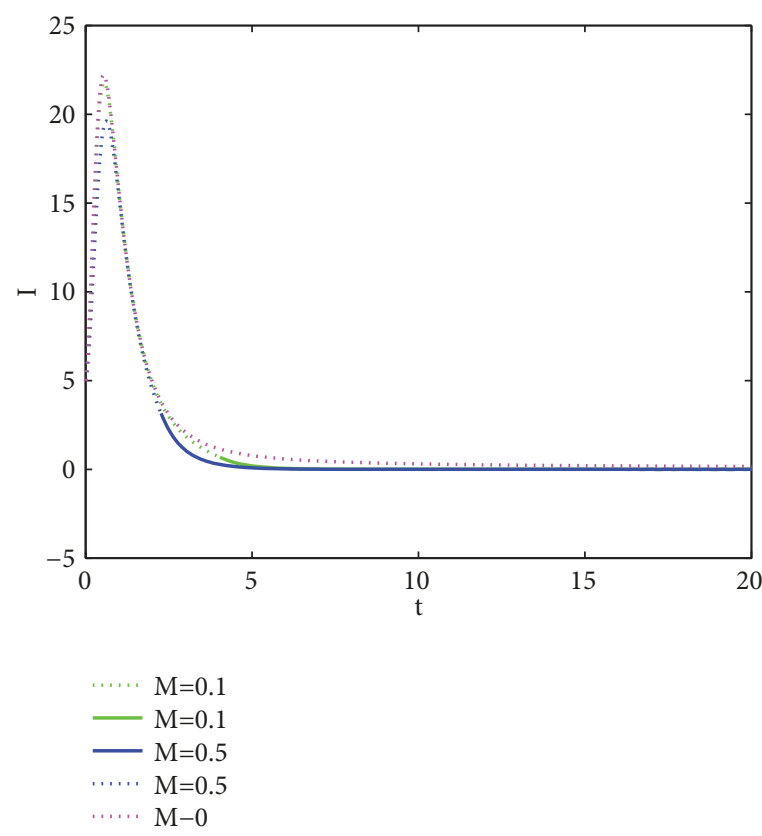

(a) $R_{0}<\min \left\{1, P_{2}\right\}$
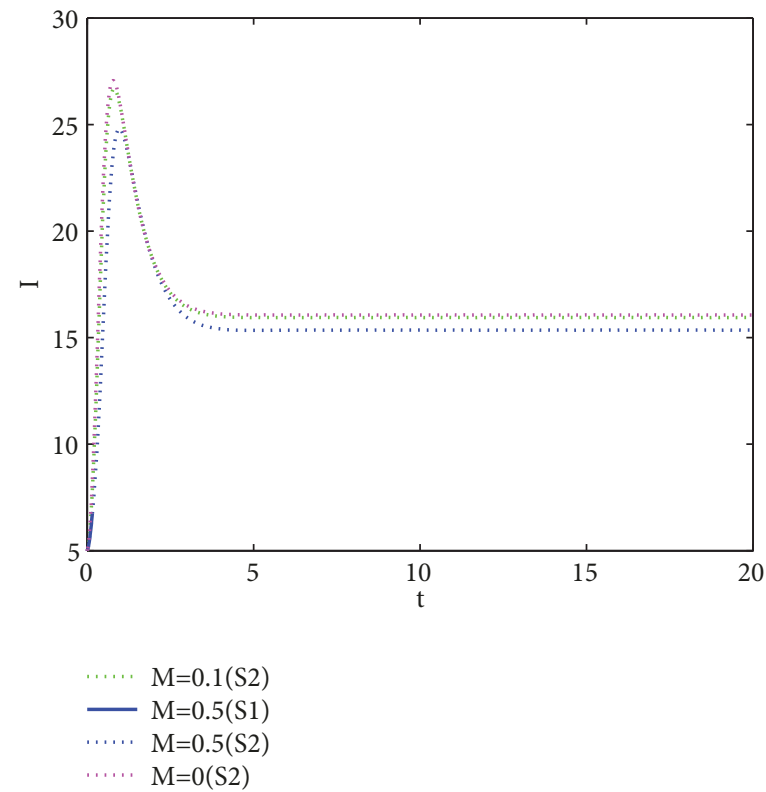

(b) $R_{0}>\max \left\{P_{2}, P_{f}\right\}$

Figure 4: Time series of the infected dairy cows. The common values of (a) and (b) used for the parameters are as follows: $w=3, d=2$, $c=0.8, \delta=4, q=1, \beta=0.2, a=2, m=0.2$. The different values of (a) and (b) are (a) $A=10$ and (b) $A=50$. The solid line or $S 1$ means that $I$ is entering system (6); the dotted line or $S 2$ indicates that $I$ is going into system (9).

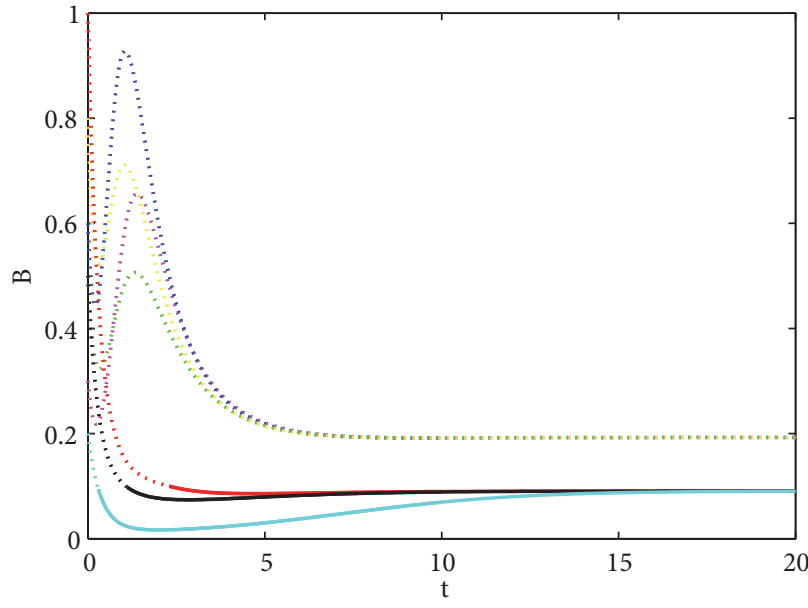

(a) The tendency of bacteria

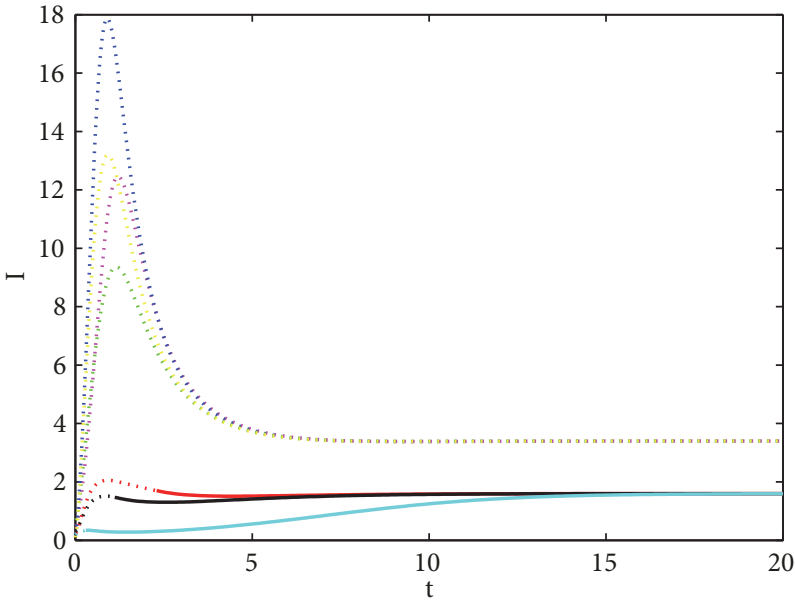

(b) The tendency of infected dairy cows

Figure 5: The common values of (a) and (b) used for the parameters are as follows: $w=3, d=2, c=0.8, \delta=4, q=1, \beta=0.2, a=2$, $m=0.1, M=0.1$, and $A=25$. Different colors are representative of different initial values, of which the dot line means that it enters system (6) and the full line means that it goes into system (9) as in Figure 4.

the final infectious size of dairy cows is tending to zero. It is to say, the disease will vanish without any measures after a certain time.

Figure 4(b) demonstrates that when $R_{0}>\max \left\{P_{2}, P_{f}\right\}$, the infected dairy cows will obtain the endemic equilibrium $E_{2}^{+}$. In the meantime, different values of $M$ have different equilibria: when $M=0.5, I_{2}^{+}=15.3531$; when $M=0.1$, $I_{2}^{+}=15.9432$; and when $M=0, I_{2}^{+}=16.0625$. This means that only if the basic reproduction number satisfies the above formula, brucellosis will never disappear and it would become epidemic disease. In addition, the more size of MID will lead to the less final infected dairy cows. It might be easy to understand that when the MID is heightened, the bacteria invading the body are diminished by immunology theory [10].

Figures 5 and 7 show that when $\min \left\{1, P_{2}\right\}<R_{0}<P_{f}$, the final size of infectious cows will be in bistable state. Figure 5 comes from Figure 2(a) when $P_{2}<R_{0}<P_{f}$ and Figure 7 


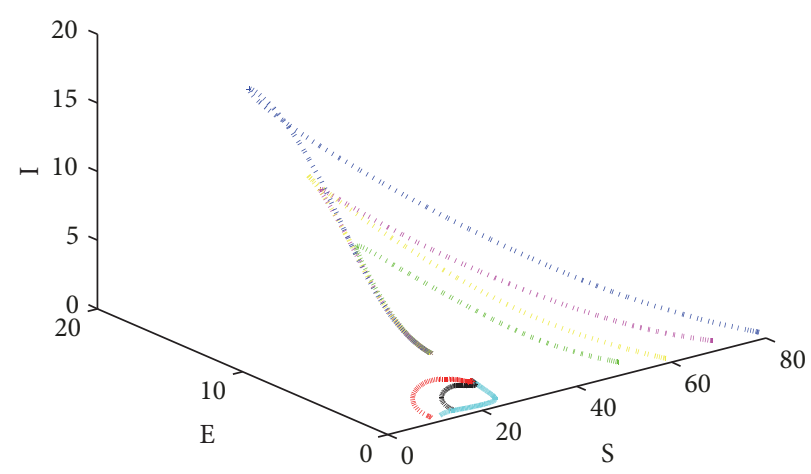

Figure 6: The tendency of $S, E$, and $I$.

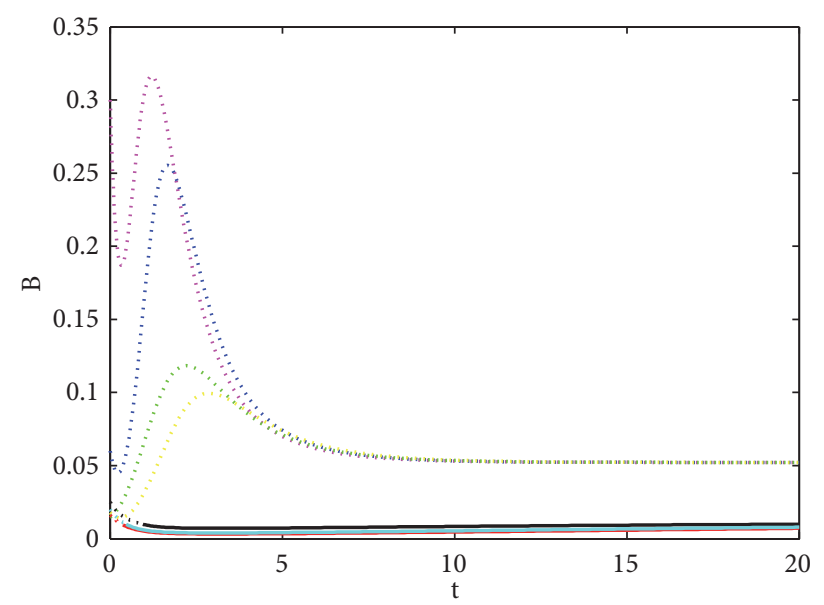

(a) The tendency of bacteria

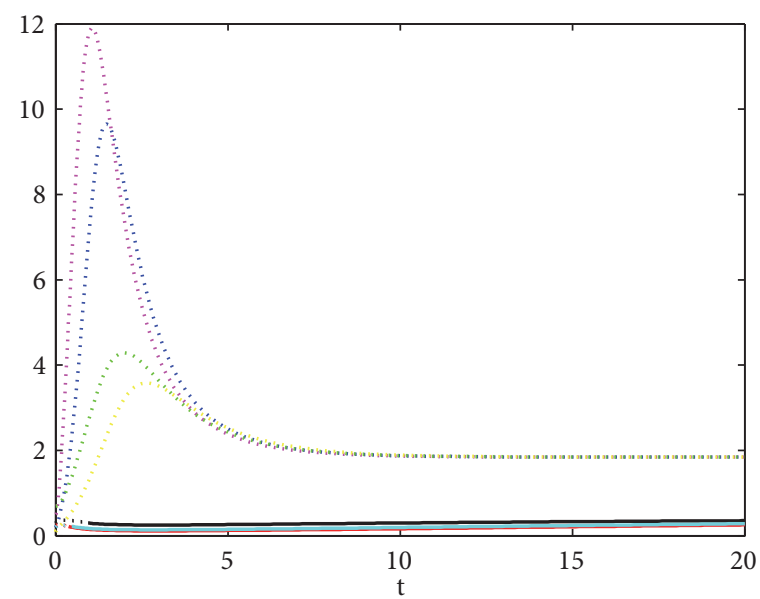

(b) The tendency of infected dairy cows

Figure 7: The common values of (a) and (b) used for the parameters are as follows: $w=3, d=2, c=0.8, \delta=4, q=1, \beta=0.2, a=2$, $m=0.05, M=0.014$, and $A=22$.

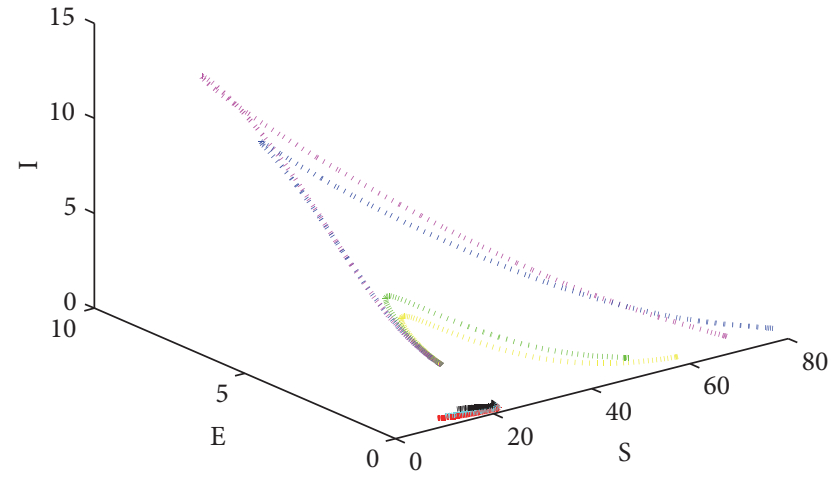

FIgURE 8: The tendency of $S, E$, and $I$.

comes from Figure 2(c) when $1<R_{0}<P_{f}$, in which Figures 5(a) and 7(a) are time series plots of the number of Brucella and Figures 5(b) and 7(b) are time series plots of the number of infected dairy cows. Figures 6 and 8 show tendency of $S$, $E, I$. From them we can see that when $R_{0}$ meets the relational expression, different initial values will eventually lead to two equilibria; one is $E_{1}$ and the other is $E_{2}^{+}\left(E_{1}<E_{2}^{+}\right)$. In biology, brucellosis will become endemic in dairy cows with two different stable states.

\section{Conclusion and Discussion}

Brucellosis is becoming more and more popular in recent years, since it has formed serious endemic disease and it cannot be thoroughly removed in northeast and northwest China. In this paper, we take account of immune influence of Brucella just like researching of cholera [12]. We build the new brucellosis model based on [22]. Compared with other studies, we are more concerned with the effects of immunology on Brucella. We just change the transmission rate from constant $\beta_{2}$ to the piecewise continuous function between the research [12] and our paper. But the mathematical results we obtain are more complicated and more biological. The original work obtained the basic reproduction number and got two equilibria and then simply analyzed the stability state. In this paper, we take immunological threshold into consideration; then we get the whole mathematical analysis of equilibria in each case and do the locally and globally stable analysis about some equilibria. The MID of Brucella has not been tested by experiment, so we get the serious discussion 
with $M>0$ and $M=0$ : when $0<M<M_{g}$ (the formation of $M_{g}$ is given in this paper), we get the forward bifurcation occurring at $R_{0}=1$ and backward bifurcation appearing at $R_{0}=P_{2}$; when $M \geq M_{g}$, it just has forward bifurcation at $R_{0}=1$; when $M=0$, the model is exactly same as in [22] and produces the forward bifurcation at $R_{0}=P_{2}$. By mathematical analysis of them, we can get the global stability $E_{0}$ when $R_{0}<1$ and a global stability $E_{1}$ when $R_{0}>1$. The character of $E_{1,2}^{+}$is given by numerical simulations. From that, we know it will be in bistable state when the basic reproduction number demands $\max \left\{1, P_{2}\right\}<R_{0}<P_{f}$. We make the basic reproduction number as the measuring standard in order to give suggestions of the brucellosis prevention [30, 31]. While the basic reproduction number is less than 1 , brucellosis is becoming a disease-free and bacteria-free stable state. After a certain time, brucellosis will be removed. And while the basic reproduction number is more than 1 by recording to different relationship about $M, M_{g}$ and 0 , the brucellosis will be endemic disease which will hold a stable state with variant values of infected dairy cows. Using the analysis of this paper, we can reduce the number of infected dairy cows to prevent the spreading of epidemic disease, which can even obtain zero by adjusting the size of the basic reproduction number.

Refs. [20, 21] using Holling - II functional response both show that MID is vital for the spread of cholera. Reducing the pathogen density to under the MID in an aquatic reservoir would be more effective measure to control cholera. Similarly, in order to control and prevent the spreading of brucellosis, we can place an emphasis on the incident rate because of the existence of MID. If we can adjust the initial value of Brucella to be below the MID and constrain growth and reproduction conditions of Brucella in environment, then we can obtain the incidence rate of indirect transmission of Brucella to susceptible cow to be zero all the time and the ultimate scale of brucellosis will decrease and even disappear.

In further planning, the incidence term $\alpha(B)$ can be used in the formation by a Holling - II response which is more reasonable in biology than the linear form in this paper. In addition, we have not yet captured and processed the real data about brucellosis of dairy cows to get more effective conclusions. That is what we are going to do next. Furthermore, the real spreading of brucellosis is via multiple zones rather than one zone and the multizone patchy model can describe the transmission in multiple regions. Hence, with considering the network models $[32,33]$ or reactiondiffusion equations [34-38], conclusions can accord well with practice even more.

\section{Data Availability}

No data were used to support this study.

\section{Conflicts of Interest}

The authors declare that they have no conflicts of interest.

\section{Acknowledgments}

The project is funded by the National Natural Science Foundation of China under Grants (11671241, 11601292, 41875097, and 11501338), Program for the Outstanding Innovative Teams (OIT) of Higher Learning Institutions of Shanxi, Natural Science Foundation of Shanxi Province Grant no. 201801D221003, and High-Level Talent Project of Jiangsu Province (Six Talent Peaks and Grant no. JNHB-071).

\section{References}

[1] M.-L. Boschiroli, V. Foulongne, and D. O'Callaghan, "Brucellosis: a worldwide zoonosis," Current Opinion in Microbiology, vol. 4, no. 1, pp. 58-64, 2001.

[2] M.-J. Corbel, "Brucellosis: an overview," Emerging Infectious Diseases, vol. 3, no. 2, pp. 213-221, 1997.

[3] M.-J. Corbel, Brucellosis in Humans and Animals, World Health Organization, 2006.

[4] T. Akhvlediani, C. T. Bautista, N. Garuchava et al., "Epidemiological and clinical features of brucellosis in the country of Georgia," PLoS ONE, vol. 12, no. 1, Article ID e0170376, 2017.

[5] B. G. Mantur and S. K. Amarnath, "Brucellosis in India-a review," Journal of Biosciences, vol. 33, no. 4, pp. 539-547, 2008.

[6] T. Barbier, F. Collard, A. Zúñiga-Ripa et al., "Erythritol feeds the pentose phosphate pathway via three new isomerases leading to D-erythrose-4-phosphate in Brucella," Proceedings of the National Acadamy of Sciences of the United States of America, vol. 111, no. 50, pp. 17815-17820, 2014.

[7] E. Moreno and I. Moriyón, "Brucella melitensis: a nasty bug with hidden credentials for virulence," Proceedings of the National Acadamy of Sciences of the United States of America, vol. 99, no. 1, pp. 1-3, 2002.

[8] B. Aïnseba, C. Benosman, and P. Magal, "A model for ovine brucellosis incorporating direct and indirect transmission," Journal of Biological Dynamics, vol. 4, no. 1, pp. 2-11, 2010.

[9] S. Singh, P. Chandra, and J. B. Shukla, "Modeling and analysis of the spread of carrier dependent infectious diseases with environmental effects," Journal of Biological Systems, vol. 11, no. 3, pp. 325-335, 2003.

[10] K.-M. Murpiiy, P. Travers, and M. Walport, Janeway's Immunobiology, Garland Science, New York, NY, USA, 7th edition, 2007.

[11] A. F. Brouwer, M. H. Weir, M. C. Eisenberg, R. Meza, and J. N. S. Eisenberg, "Dose-response relationships for environmentally mediated infectious disease transmission models," PLoS Computational Biology, vol. 13, no. 4, Article ID e1005765, 2017.

[12] J. D. Kong, W. Davis, X. Li, and H. Wang, "Stability and sensitivity analysis of the iSIR model for indirectly transmitted infectious diseases with immunological threshold," SIAM Journal on Applied Mathematics, vol. 74, no. 5, pp. 1418-1441, 2014.

[13] G.-G. Jorge and R. Naulin, "Analysis of a model of bovine brucellosis using singular perturbations," Journal of Mathematical Biology, vol. 33, no. 2, pp. 211-223, 1994.

[14] M. Li, G. Sun, W. Zhang, and Z. Jin, "Model-based evaluation of strategies to control brucellosis in China," International Journal of Environmental Research and Public Health, vol. 14, no. 3, article 295, 2017.

[15] M. Li, G. Sun, J. Zhang et al., "Transmission dynamics and control for a brucellosis model in Hinggan League of Inner Mongolia, China," Mathematical Biosciences and Engineering, vol. 11, no. 5, pp. 1115-1137, 2014

[16] G.-Q. Sun and Z.-K. Zhang, "Global stability for a sheep brucellosis model with immigration," Applied Mathematics and Computation, vol. 246, pp. 336-345, 2014. 
[17] J. Zinsstag, F. Roth, D. Orkhon et al., "A model of animal-human brucellosis transmission in Mongolia," Preventive Veterinary Medicine, vol. 69, no. 1-2, pp. 77-95, 2005.

[18] Q. Hou, X. D. Sun, J. Zhang, Y. J. Liu, Y. M. Wang, and Z. Jin, "Modeling the transmission dynamics of sheep brucellosis in Inner Mongolia Autonomous Region, China," Mathematical Biosciences, vol. 242, no. 1, pp. 51-58, 2013.

[19] M.-T. Li, G.-Q. Sun, Y.-F. Wu, J. Zhang, and Z. Jin, "Transmission dynamics of a multi-group brucellosis model with mixed cross infection in public farm," Applied Mathematics and Computation, vol. 237, no. 11, pp. 582-594, 2014.

[20] J. D. Kong, W. Davis, and H. Wang, "Dynamics of a cholera transmission model with immunological threshold and natural phage control in reservoir," Bulletin of Mathematical Biology, vol. 76, no. 8, pp. 2025-2051, 2014.

[21] R. I. Joh, H. Wang, H. Weiss, and J. S. Weitz, "Dynamics of indirectly transmitted infectious diseases with immunological threshold," Bulletin of Mathematical Biology, vol. 71, no. 4, pp. 845-862, 2009.

[22] J. Nie, G.-Q. Sun, X.-D. Sun et al., "Modeling the transmission dynamics of dairy cattle brucellosis in Jilin Province, China," Journal of Biological Systems, vol. 22, no. 4, pp. 533-554, 2014.

[23] W. D. Wang, "Backward bifurcation of an epidemic model with treatment," Mathematical Biosciences, vol. 201, no. 1-2, pp. 58-71, 2006.

[24] O. Y. Sharomi, M. A. Safi, A. B. Gumel, and D. J. Gerberry, "Exogenous re-infection does not always cause backward bifurcation in TB transmission dynamics," Applied Mathematics and Computation, vol. 298, pp. 322-335, 2017.

[25] P. van den Driessche and J. Watmough, "Reproduction numbers and sub-threshold endemic equilibria for compartmental models of disease transmission," Mathematical Biosciences, vol. 180, pp. 29-48, 2002.

[26] L. Li, C.-H. Wang, S.-F. Wang et al., "Hemorrhagic fever with renal syndrome in China: mechanisms on two distinct annual peaks and control measures," International Journal of Biomathematics, vol. 11, no. 2, Article ID 1850030, 2018.

[27] H. B. Guo, M. Y. Li, and Z. S. Shuai, "A graph-theoretic approach to the method of global Lyapunov functions," Proceedings of the American Mathematical Society, vol. 136, no. 17, pp. 2793-2802, 2008.

[28] A. Korobeinikov, "Lyapunov functions and global stability for SIR and SIRS epidemiological models with non-linear transmission," Bulletin of Mathematical Biology, vol. 68, no. 3, pp. 615-626, 2006.

[29] J. P. LaSalle, The Stability of Dynamical Systems, SIAM, Philadelphia, Pa, USA, 1976.

[30] S. Deqiu, X. Donglou, and Y. Jiming, "Epidemiology and control of brucellosis in China," Veterinary Microbiology, vol. 90, no. 1-4, pp. 165-182, 2002.

[31] J. Zhang, G.-Q. Sun, X.-D. Sun et al., "Prediction and control of brucellosis transmission of dairy cattle in Zhejiang Province, China," PLoS ONE, vol. 9, no. 11, Article ID e108592, 2014.

[32] L. Li, J. Zhang, C. Liu, H.-T. Zhang, Y. Wang, and Z. Wang, "Analysis of transmission dynamics for Zika virus on networks," Applied Mathematics and Computation, vol. 347, pp. 566-577, 2019.

[33] X.-X. Zhan, C. Liu, G. Zhou et al., "Coupling dynamics of epidemic spreading and information diffusion on complex networks," Applied Mathematics and Computation, vol. 332, pp. 437-448, 2018.
[34] G.-Q. Sun, M. Jusup, Z. Jin, Y. Wang, and Z. Wang, "Pattern transitions in spatial epidemics: Mechanisms and emergent properties," Physics of Life Reviews, vol. 19, pp. 43-73, 2016.

[35] L. Li, "Patch invasion in a spatial epidemic model," Applied Mathematics and Computation, vol. 258, pp. 342-349, 2015.

[36] G. Bocharov, A. Meyerhans, N. Bessonov, S. Trofimchuk, and V. Volpert, "Interplay between reaction and diffusion processes in governing the dynamics of virus infections," Journal of Theoretical Biology, vol. 457, pp. 221-236, 2018.

[37] G.-Q. Sun, C.-H. Wang, L.-L. Chang, Y.-P. Wu, L. Li, and Z. Jin, "Effects of feedback regulation on vegetation patterns in semi-arid environments," Applied Mathematical Modelling: Simulation and Computation for Engineering and Environmental Systems, vol. 61, pp. 200-215, 2018.

[38] S. Islam and R. Zaman, "A computational modeling and simulation of spatial dynamics in biological systems," Applied Mathematical Modelling, vol. 40, no. 7-8, pp. 4524-4542, 2016. 


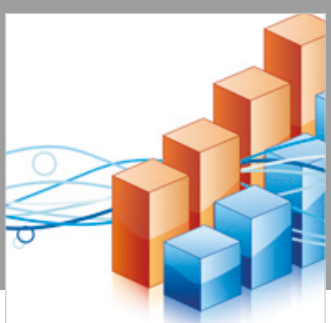

Advances in

Operations Research

\section{-n-m}
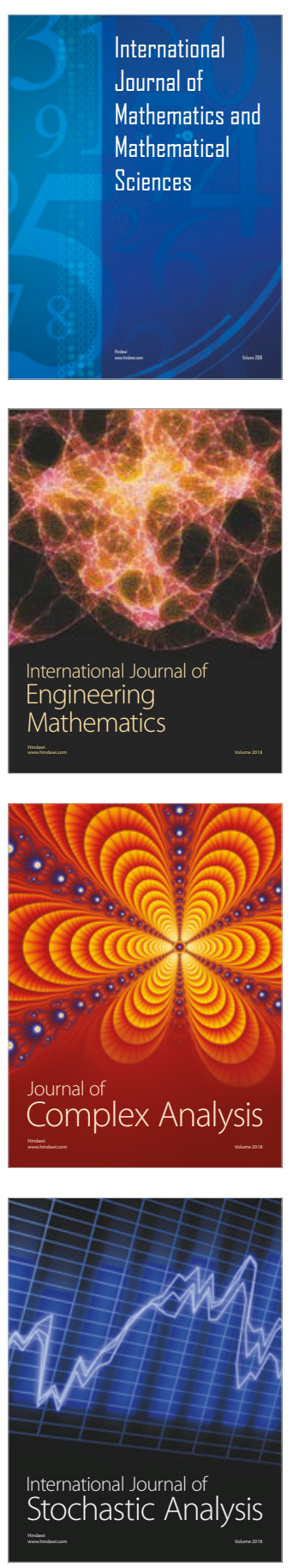
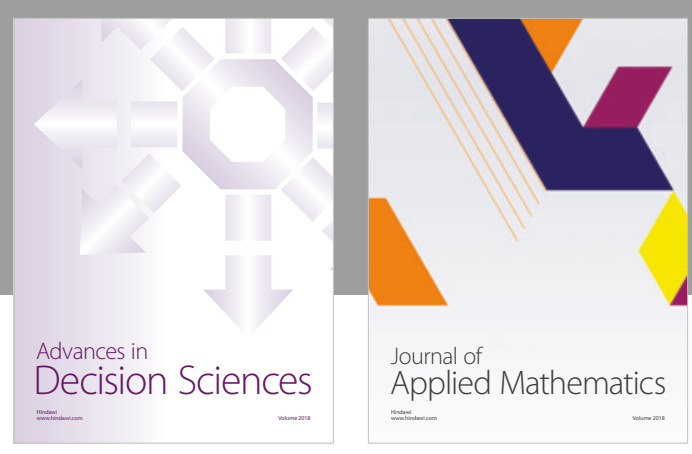

Journal of

Applied Mathematics
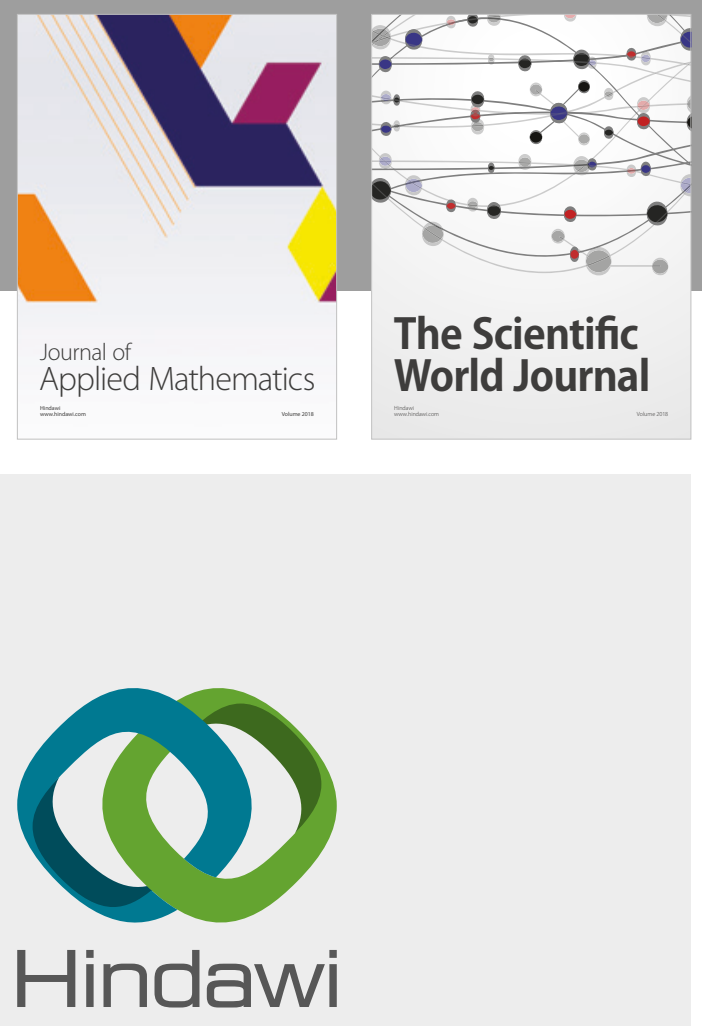

Submit your manuscripts at

www.hindawi.com

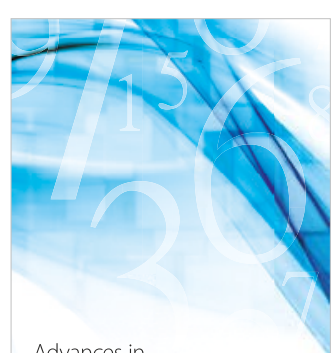

Advances in
Numerical Analysis
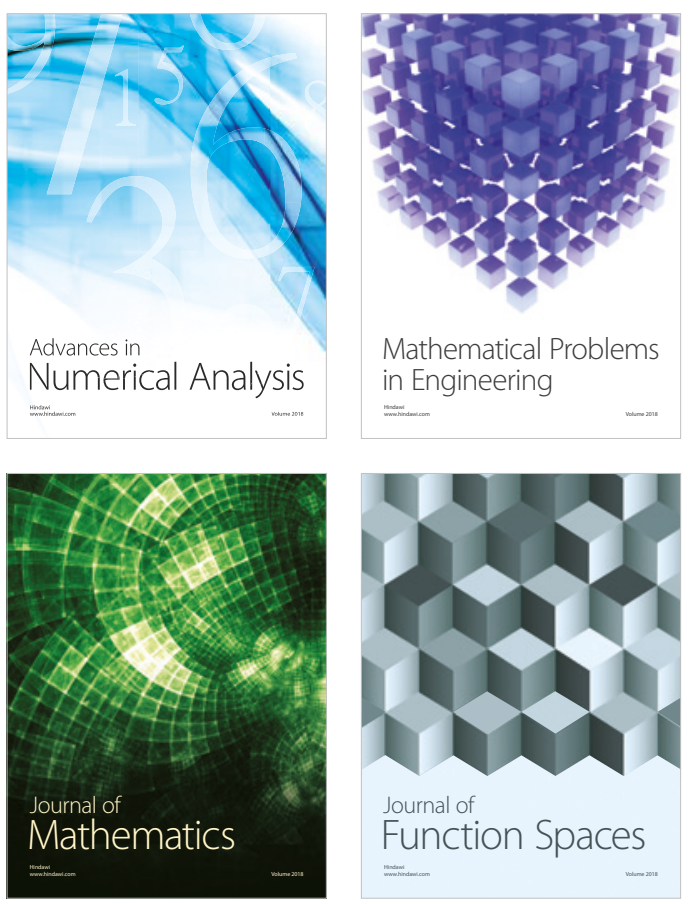

Mathematical Problems in Engineering

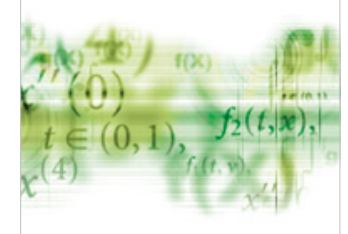

International Journal of

Differential Equations

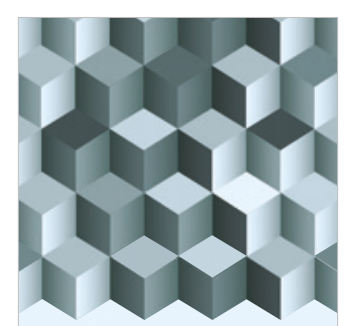

Journal of

Function Spaces
The Scientific

World Journal

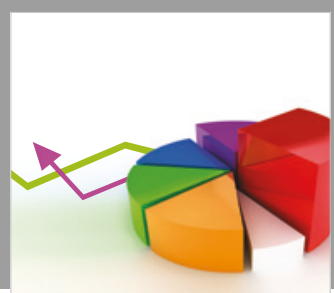

Journal of

Probability and Statistics
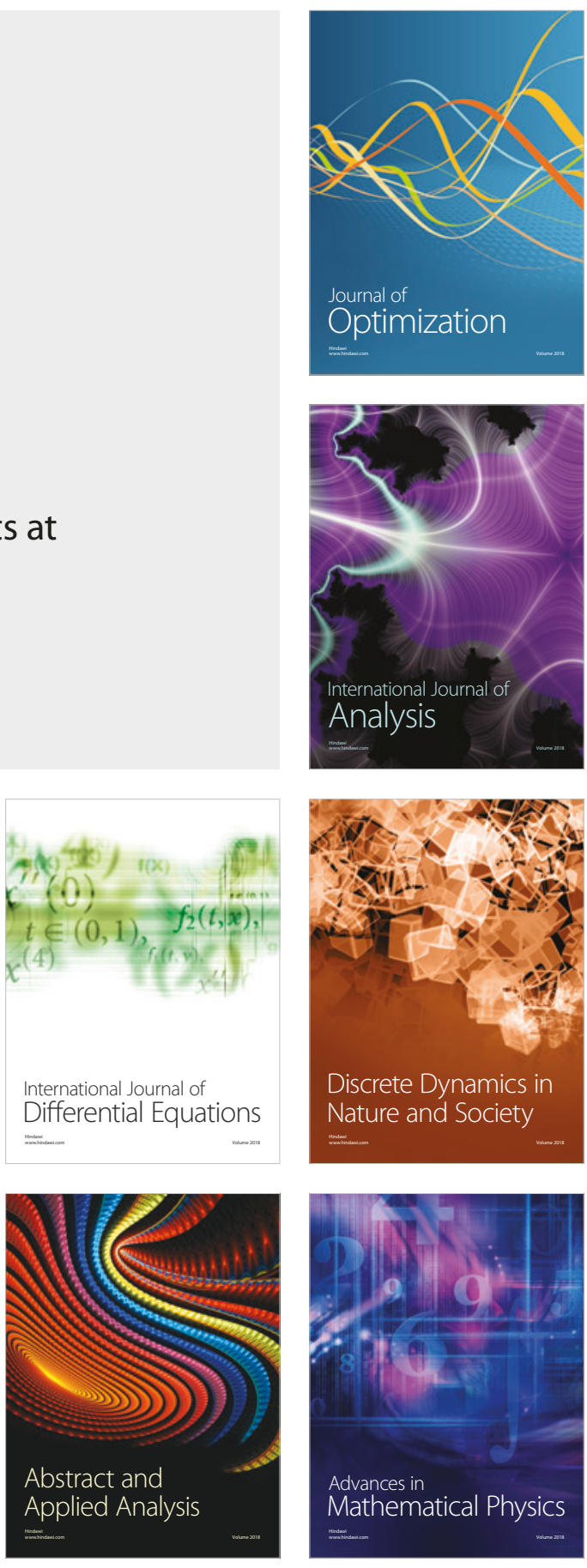